\title{
COVID-19: A Systematic Review of the Mechanisms of Injury to the Central Nervous System This Far
}

Laura Loeb'

Paulo Afonso Mei ${ }^{1,2}$

\section{ABSTRACT}

2019-nCoV (COVID-19) is a single-stranded, positive-sense, encapsulated betacoronavirus, and continues to spread throughout the world. Various neurological symptoms, ranging from mild to fatal, are continuously reported, raising the importance of a better understanding of the mechanisms through which 2019-nCoV invades and harms the central nervous system (CNS). A systematic review was performed using 18 articles and it was concluded that there is strong evidence supporting the link between 2019-nCoV and nervous damage, and that there are at least three means by which it may directly enter the CNS, and three by which it may cause indirect harm to the CNS.

Key Words: SARS-CoV-2, COVID-19, 2019-nCoV, central nervous system, neurological aspects, mechanisms of invasion, mechanisms of injury.

\section{INTRODUCTION}

On December of 2019, a distinct form of coronavirus was reported in Wuhan, China, after several people reported to health facilities with severe pneumonia of unknown origin. Genetic analysis of viral particles obtained from respiratory tract samples from affected patients revealed the existence of a new betacoronavirus, dados da produção named 2019 novel coronavirus (2019$\mathrm{nCoV}$ ) or severe acute respiratory syndrome coronavirus type 2 (SARS-CoV-2), given its genetic similarity to SARSCoV, which had its outbreak in 2002 [1].

2019-nCoV belongs to the Coronaviridae family, composed of positive-sense, single stranded enveloped, RNA viruses which exhibit a crown-like appearance due to the expression of certain surface proteins [1]. Besides 2019-nCoV, which belongs to the $B$ genera, six other coronavirus strains are known to infect humans: HCoV-
NL63 and HCoV-229E (from the B genera), and HCoVOC43, HCOV-HKU1, SARS-CoV, and MERS-CoV, (from the $B$ genera). In addition, like SARS-CoV, 2019-nCoV expresses spike proteins on its envelope that bind to the angiotensin conversion enzyme receptors type 2 (ACE2), expressed in various tissues.

Although many patients display only respiratory signs of infection, mild and severe neurological symptoms are becoming increasingly recognized as an effect of 2019nCoV: one study reported the development of neurological symptoms or conditions in more than one third of infected patients [3], raising our attention on the virus' potential to harm the central nervous system (CNS), worsen pre-existing neurological conditions, or lead to long term neurological damage. With these concerns under consideration, we performed a systematic review of the proposed mechanisms of invasion and injury to the CNS by 2019-nCoV.

${ }^{1}$ Faculdade de Medicina São Leopoldo Mandic, Campinas, Brazil.

${ }^{2}$ University of Campinas - UNICAMP, Campinas, Brazil 
We searched for open-access papers published in English from 1971 to 2020. The following key-words were used: SARS-CoV-2, COVID19, 2019-nCoV, SARSCOV, MERS-CoV, or coronavirus and central nervous system, lungs, cytokine storm, neurotropism, neurological symptoms, neurological complications, neurological disorders, injuries, and invasion mechanisms. The following databases were used: PubMed, PubMed Central, EMBASE, and Google Scholar. More than 30 articles were accessed, and 18 were included.
Neurological symptoms and disorders caused by 2019-nCov

There seems to be a strong association between 2019-nCoV and the development of mild and serious neurological complications [3]. Although it is not within the scopes of this review to go over details about clinical findings associated to 2019-nCoV, we find it important for the reader to come across some examples before delving into the mechanisms of injury (Figure 1).

Figure 1. The virus can enter the CNS by neuronal retrograde or axonal path, hematogenous path or immune path. Indirect injury can be due to the immune and inflammatory responses, to hypoxia, or to vascular alterations.

\section{COVID-19 MECHANISMS OF INJURY TO THE CNS}

\section{DIRECT}

\section{Virus enters CNS by:}

Neuronal retrograde/ axonal path

\section{$>$ Hematogenous path}

Immune path
INDIRECT

Caused by:

$>$ Immune/ inflammatory response

$>$ Hypoxia

$>$ Vascular lesions
A study performed in Wuhan by Mao et al. [3] noted that in a series of 214 confirmed cases (median age of 52.7, 6 females: 4 males ratio), 78 (36.4\%) developed neurological symptoms such as dizziness (36 patients $16.8 \%$ ), headache (28 patients - $13.1 \%$ ), skeletal muscle injury (23 patients $-10.7 \%)$, altered consciousness (16 patients $-7.5 \%)$ dysgeusia (12 patients $-5.6 \%)$, anosmia patients - 7.5\%), dysgeusia (12 paients $-5.6 \%)$, anosmia (11 patients $-5.1 \%)$, stroke (6 patients $-2.8 \%)$, neuralgia $(5$ patients $-2.3 \%)$, unspecified visual impairment (
patients $-1.4 \%)$, and ataxia and seizure (1 patient male with aseptic encephalitis [5], and PanizMandolfi et al. reported the presence of 2019-nCov specimens in neural and capillary endothelial cells from frontal lobe tissue obtained in post-mortem examination of an infected 78-year-old male [6].

\section{Mechanisms of Injury}

We interpret that 2019-nCoV's mechanisms of injury to the CNS may be direct, once evidence shows that vira particles can enter the CNS $[6,7,8,9]$ or indirect, when the injury is caused by exacerbated inflammation in the CNS following viral infection, by the detrimental effects of hypoxia caused by lung tissue damage following vira infection, or to other systemic disturbances [11]. It is reasonable, however, to assume that the injury may result from a combination of components.

\section{Direct Injury}

It is inferred that direct injury occurs when 2019$n C o V$ specimens are present inside the nervous system. Neuro-invasiveness by coronaviruses has long been documented. In 1981, Gerdes et al. [7] reported autopsies from two Multiple Sclerosis patients, where coronaviruses were isolated and exhibited cross-reactivity with OC43.

There is more than one proposed mechanism through which 2019-nCoV could enter the nervous system: the neuronal retrograde route (in which neurons serve as bridges for viral entry), the hematogenous route (in which viruses in the blood are able to penetrate the blood-brain-barrier and infect CNS structures), and finally, the immune mediated entry (in which immune cells serve as carriers of the virus into the CNS).

Neuronal retrograde path: Baig et al. [8] propose in the virus reaches the brain through neurons of the olfactory bulb, which is supported by findings of anosmia and hyposmia in a considerable portion of patients. In addition an experimental study by Desforges et al. [9] with mice showed that intranasal infection with $\mathrm{HCOV}-\mathrm{OC} 43$ and SARS-CoV led to the invasion of the nervous system via the nasal epithelium, followed by neural dissemination, which support the involvement of the olfactory nerve and the early onset of anosmia as an indicator of neural invasion by the virus. Moreover, previous works with rats seem to support this line of thought - In 1990, Perlman
Evans and Afifi [10] performed unilateral surgical ablation of the olfactory bulb in mice infected with the JHM strain of the Mouse Hepatitis Virus, a member of the coronavirus family, by nasal inoculation. Autopsy and analysis by in situ hybridization and emulsion radiography of sagittal slices showed that the ablation partially prevented infection, as CNS sites proximal to the olfactory connections did not show viral spread in the hemisphere ipsilateral to the ablation, although the virus was detected in more distal sites, like the mammillary nuclei and the limbic cortex, suggesting that the virus reached reached ither via contralateral connections with the preserved olfactory bulb, and/or by the trigeminal nerve.

Hematogenous path: Another probable route for 2019-nCoV into the nervous system is the hematogenous pathway. Paniz-Mondolfi et al., using electronic microscopy, observed viral particles in neural and capillary endothelial cells from frontal lobe tissue obtained from post-mortem examination of a 74-year-old male who expired due to 2019-nCoV complications proposed hematogenous pathway a fer infecting the proposed hematogenous pathway, after infecting the bloodstream, viral particles would invade endothelial cells of the blood-brain-barrier and epithelial cells of the brain-cerebral-spinal-fluid barrier and choroid plexus. The hypothesis that the blood provides an entryway into the CNS is also supported by the fact that the epithelia present in the blood-brain barrier express ACE2 receptors, which serve as a link to the viral spike protein [11] indicating that the penetration of the blood-brain-barrier by 2019$\mathrm{nCoV}$ is theoretically possible.

mmune path: A third possibility, supported by the worsening of neurological symptoms observed in patients undergoing a cytokine storm, is the immune-mediated entry of 2019-nCoV into the CNS. In this pathway, instead of binding directly to ACE2 receptors in the blood vessels, the virus is carried into the CNS inside immune or inflammatory cells, which can penetrate themselves the blood-brain barrier, and later release the virus in the CSF and the brain tissue [11]

\section{Indirect Injury}

Injury mediated by immune and inflammatory cells: Neurotropic viruses such as 2019-nCoV can induce an exacerbated pro-inflammatory state in the CNS, mediated by immune cells, cytokines (chemokines and others), radicals, and other agents $[11,14]$. Although these factors do serve protective and regenerative purposes in the CNS, 
their exacerbated expression is also associated to most severe cases of 2019-nCoV and to cases which develop neurologicalcomplications $[11,12,14]$. Followinginfection of mice with JHM virus, another neurotropic pathogen, significant chemokine signaling, demyelination injuries in the brain and spinal cord, and axonal degeneration were observed [15].

Moreover, Interleukin-10 elevation before complete pathogen clearance led to persistent inflammation of the CNS, therefore, control of the inflammatory response is crucial to the resolution of the viral infection [17] Bohmwald et al. [12] confirm that, upon infection with coronavirus, large amounts of interleukins (IL): IL-6, IL-

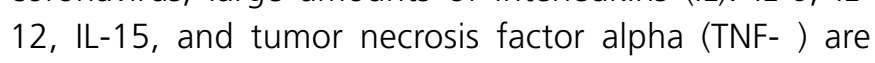
detected. IL-6 is an imperative component of the cytokine storm, which is strongly linked to the most severe cases of infection by 2019-nCoV. Furthermore, Yang et al.

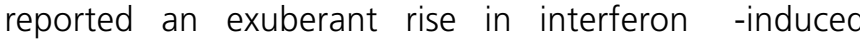
protein 10 (IP-10 or CXCL10, a T cell chemoattractant chemokine), monocyte chemotactic protein-3 (MCP3) and IL-1 receptor antagonist (IL-1 ra) levels in severe cases of 2019-nCoV infection [13]. Skinner et al. also indicated that considerable chemokine signaling from infected cells occurs following viral infection, and that IP10 levels increases drastically and can be responsible for viral-induced demyelination [15]

Injury caused by hypoxia: Hypoxic injury to the CNS can occur due to 2019-nCoV's extensive damage to the lungs and impairment of gas exchanges [11]. A histopathologic examination of the lung tissue from a 72-year-old man with 2019-nCoV revealed diffuse alveolar damage, pneumocyte type II hyperplasia, intra-alveolar fibrinous exudate, loose interstitial fibrosis, chronic inflammatory infiltrates and damaged, desquamated cells within the alveolar space [18]. When gas exchanges are sufficiently impaired due to these severe histo- and physiological alterations, a state of hypoxia is established, leading to metabolic acidosis, which may cause cerebral vasodilation, swelling of brain cells, interstitial edema, and obstruction of cerebral blood flow [11]. The interstitia edema can lead to intracranial hypertension, which can subsequently lead to gradual deterioration of brain function and stroke [11]

Vascular originated injury: Binding of 2019-nCoV to ACE2 receptors of the blood vessels leads to drastic elevation of the blood pressure and increases the risk of cerebral hemorrhage. Moreover, the expression of ACE2 in the capillary vessel epithelium allows the virus to harm and evade the blood-b vascular damage inside the CNS [11]

\section{Discussion}

It is clear that 2019-nCoV has an alarming potential to affect the nervous system, and to cause serious illnesses such as seizures, hemorrhagic or ischemic strokes, encephalitis, meningitis, Guillain-Barré Syndrome, and more than one third of infected patients developed neurological symptoms, and that 2019-nCov has currently infected more than 4 million people and accounts for more than 280,000 deaths worldwide [15], it is crucial for neurologists and other health professionals, as well as health-care systems and facilities to prepare and be ready to diagnose and treat short-term neurological complications, to implement follow up protocols - once investigate preventions and treatrent strages. For that, a better understanding of the basic virology and pathophysiology of the virus is crucial for optimization of prevention, care and development of efficacious therapies.

Although there are different proposed pathways by which 2019-nCoV may harm the CNS, it remains unclear if one theoretical entryway prevails over the others, and if they may take place concomitantly. Further investigation on how the virus is able to penetrate de boundaries of the CNS is, thus, essential.

In addition, neurological complications were more prevalent in severe cases than in mild ones, reinforcing how imperative neurological assessment is in 2019nCoV patients, especially in those with underlying comorbidities, which tend to manifest more lifethreatening complications.

Conclusion

This review went briefly over the neurological symptoms and conditions caused by 2019-nCoV, and then discussed the direct and indirect ways through which this virus can damage the nervous system. The authors reckon that there is a strong link between various neurolical conditions and $2019-\mathrm{COV}_{1}$ that are various mechanisms through which the virus can disrupt the CNS, and extensive research and preparation is still needed to better understand and minimize the neurological damages caused by 2019-nCoV.

\section{REFERENCES}

[1] ZHU, Na; ZHANG, Dingyu; WANG, Wenling; et al. A Nove Coronavirus from Patientswith Pneumoniain China, 2019. New England Journal of Medicine, v. 382, n. 8, p. 727-733, 2020

2) BALTIMORE, D. Expression of animal virus genomes. Bacteriological Reviews, v. 35, n. 3, p. 235-241, 1971

[3] MAO, Ling; JIN, Huijuan; WANG, Mengdie et al. Neurologic Manifestations of Hospitalized Patients With Coronavirus Disease 2019 in Wuhan, China. JAMA Neurology, 2020. Disponível em: <https://jamanetwork.com/journals/jamaneurology/ fullarticle/2764549>. Acesso em: 25 abr. 2020.

[4] NATH, Avindra, Neurologic Complications 14] NATH, Avindra, Neurologic Complications $\begin{array}{lllll}\text { of Coronavirus Infections, American Academy } \\ \text { of Neurology, v. } 94 & \text { n. } 21 & \text { p. } & 1-2,2020 .\end{array}$

[5] MORIGUCHI, Takeshi; HARII, Norikazu; GOTO, Junko; et al. A first case of meningitis/encephalitis associated with SARS-Coronavirus-2. International Journal of Infectious Diseases, v. 94, p. 55-58, 2020

[6] PANIZ-MONDOLFI, Alberto; BRYCE, Clare; GRIMES, Zachary; et al. Central Nervous System Involvement by Severe Acute Respiratory Syndrome Coronavirus $₫ 2$ (SARS CoV-2). Journal of Medical Virology, p. jmv.25915, 2020.

[7] GERDES, J. C.; KLEIN, I.; DEVALD, B. L.; et al. Coronavirus isolates SK and SD from multiple sclerosis patients are serologically related to murine coronaviruses A59 and JHM and human coronavirus $\mathrm{OC} 43$, but not to human coronavirus 229E. Journal of Virology, v. 38, n. 1, p. 231-238, 1981

[8] BAIG, Abdul Mannan; KHALEEQ, Areeba; ALI, Usman; et al. Evidence of the COVID-19 Virus Targeting the CNS: Tissue Distribution, Host-Virus Interaction, and Proposed Neurotropic Mechanisms. ACS Chemical Neuroscience, v. 11, n. 7, p. 995- 998, 2020

[9] Desforges M, Le Coupanec A, Dubreau P, Bourgouin A Lajoie L, Dubé M, Talbot PJ. Human Coronaviruses and other respiratory viroses: nderestimated opportunistic pathogens

PERLMAN

Stanley(7)

11] WU, Yeshun; XU, Xiaolin; CHEN, Zijun; et al. Nervous system involvement after infection with COVID-19 and other coronaviruses. Brain, Behavior, and Immunity, p. S0889159120303573, 2020

12] BOHMWALD, Karen; GÁLVEZ, Nicolás M. S.; RíOS, Mariana; et al. Neurologic Alterations Due to Respiratory Virus Infections. Frontiers in Cellular Neuroscience, v. 12, Virus Infections. Frontiers in Cellular Neuroscience, v. 12, 2018. Disponivel em: <https./Www.ncbi.nlm.nin.gov/ pmc/articles/PMC6212673/>. Acesso em: 5 maio 2020 [13] YANG, yang et al, Exuberat elevation of IP-10, MCP-3 and IL-1rA during SARSCOV-2 infection is associated with disease severity and fatal outcome. Disponível em: https://doi.org/1

14] LUCAS, Sian-Marie et al. The role of Inflammation in CNS Injuryand Disease. British Journal of Pharmacologyv. 147.2006.

[15] SKINNER, Dominic et al. Chemokine CXCL10 and Coronavirus-induced Neurologic Disease. Viral Immunology [16] TRANDEM, Kathryn et al. Highly Activated Cytotoxic CD8 T Cells Express Protective IL-10 at the Peak of Coronavirus-Induced Encephalitis. The Journal of Immunology. V.186. 2011. doi:10.4049/jimmunol.1003292.

[17] ZHANG, Huilan et al. Histopathologic Changes and SARS-COV-2 Immunostaining in The Lung of a Patient With COVID-19. Annals of Medicine v. 172 n.9. 2020. Doi: $\begin{array}{lllllllllllllll} & 7 & 3 & 6 & 6\end{array}$

World Health Organization

[18] WORLD HEALTH ORGANIZATION. World Health Organization Covid Dashboard. Disponível em: <https://covid19.who.int>. Acesso em: 25 abr. 2020 